\title{
Embedded Cervical Esophagogastrostomy: A Simple and Convenient Method Using a Circular Stapler After Esophagectomy for Esophageal Carcinomas
}

\author{
Sen Wu, MS, Mingyao Chen, BS, Li Wei, MS, and Zhong Chen, MS \\ Center of Thoracic Tumor, Henan Provincial People's Hospital, Zhengzhou, China
}

\begin{abstract}
Background. Cervical esophagogastrostomy is currently the most common method for esophageal reconstruction after esophagectomy. The advantages and disadvantages of hand-sewn, linear-stapled, or circular-stapled anastomoses have been subject to debate in recent years. We explored a new method of end-to-side anastomosis using a circular stapler that embeds the anastomosis and the remaining esophageal tissue into the gastric cavity to reduce the occurrence of anastomotic leakage and to prevent gastroesophageal reflux.
\end{abstract}

Methods. In 127 patients with esophageal carcinomas, end-to-side anastomoses with esophageal embedding were performed by connecting the anvil and body of the circular stapler inside the stomach before firing and embedding the anastomosis and remaining esophagus into the stomach after esophagectomy. Retrospective investigations on postoperative complications such as leakage, stricture, and gastroesophageal reflux were conducted.

Results. A total of 123 patients $(96.9 \%)$ had successful surgery, and 4 patients $(3.3 \%)$ developed anastomotic leakage, with the total morbidity of 20 of $123(16.3 \%)$ and in-hospital mortality of 1 of $123(0.8 \%)$. The incidence of stricture $(<1 \mathrm{~cm})$ affected 14 of 123 patients $(11.4 \%)$. Eight patients underwent dilatation treatment as a result of severe dysphagia (6.5\%). Half of the patients [62 of 123 $(50.4 \%)]$ experienced postoperative heartburn, 11 of 123 patients $(8.9 \%)$ experienced acid regurgitation, and 16 of 123 patients $(13.0 \%)$ experienced nocturnal cough.

(C) The Author(s) 2013. This article is published with open access at Springerlink.com

First Received: 12 February 2012;

Published Online: 5 May 2013

S. Wu, MS

e-mail: ws1213@tom.com
Conclusions. Embedded cervical esophagogastrostomy with circular stapler is a simple and convenient method, with low incidence of anastomotic leakage and a good antireflux effect.

Esophageal cancer is the eighth most common malignant tumor worldwide, and surgery is still the preferred method of treatment. ${ }^{1}$ An international survey of 269 surgeons revealed that left cervical esophagogastrostomy is one of the most common procedures used to treat esophageal cancer, and the digestive tract was most frequently restored with a gastric conduit. ${ }^{2}$ Still, esophagogastrostomy remains a challenge for the surgical treatment of esophageal cancer, with common complications that include postoperative anastomotic leakage, anastomotic stricture, and gastroesophageal reflux.,

Since the 1970s, the clinical application of mechanical anastomosis has grown in acceptance. However, the choice of hand-sewn or mechanical and end-to-side or side-to-side techniques of esophagogastrostomy is still under debate. Orringer et al. conducted a study of side-to-side esophagogastrostomy with an EndoGIA stapler (Coviden AG, Dublin, Ireland) in 114 patients and reported only 3 cases of postoperative anastomotic leakage using this method. ${ }^{5} \mathrm{~A}$ comparative study between side-to-side stapled anastomosis and hand-sewn anastomosis indicated no difference between the methods, suggesting that clinical experience was a more important consideration. ${ }^{6}$ In early studies, circular stapler use was not considered applicable to cervical esophagogastrostomy, although the comparative study of Hsu et al. demonstrated that cervical esophagogastrostomy with circular stapler had similar efficacy as hand-sewn anastomosis. ${ }^{7,8}$

At our institution, we perform end-to-side esophagogastrostomy with a circular stapler by embedding the anastomosis and the remaining esophagus into the residual 
stomach, placing it into the gastric cavity to prevent erosion by saliva, and further surrounding it by the proximal stomach to prevent gastroesophageal reflux. Our study used a retrospective analysis to evaluate the efficacy of this embedded method of cervical esophagogastrostomy with a circular stapler.

\section{MATERIALS AND METHODS}

\section{Patient Information}

The protocol for this study was reviewed and approved by the institutional review board at the Henan Provincial People's Hospital, in Zhengzhou, China, and complies with the 2004 revision of the Declaration of Helsinki. We chose patients with esophagus cancer at tumor stages T3 or T4 or with unresectable lymph nodes as indications of preoperative chemoradiation therapy. Of the patients receiving esophagectomy and excluding those who received preoperative radiotherapy, chemotherapy, or exploratory surgery, a total of 127 patients with esophagus cancer had cervical esophagogastrostomy from March 2008 to September 2010: $79(62.2 \%)$ men and 48 (37.8\%) women, with a mean age of $59.1 \pm 7.0$ years. All patients were diagnosed by fibergastroscopic biopsy, with 1 case of small cell carcinoma, 3 cases of adenocarcinoma, and the rest with squamous carcinoma. Barium meal tests, computed tomography (CT) scans, and color Doppler ultrasonography were used to confirm diagnosis. A total of 9 patients had cancer located in the upper thoracic portion, 81 in the middle thoracic portion, and 37 in the lower thoracic portion. The patients were staged according to the tumor, node, metastasis (TNM) Classification of Malignant Tumors, 7th edition (International Union Against Cancer, 2009), with 7 cases staged at IA, 9 at stage IB, 17 at stage IIA, 43 at stage IIB, 27 at stage IIIA, 21 at stage IIIB, and 3 at stage IIIB. Because middle and lower esophageal carcinomas are more common, left thoracotomy with two-field lymphadenectomy was sufficient in most cases to access mediastinal and perigastric lymph nodes. If cervical lymph node metastases were diagnosed before surgery, chemoradiotherapy might have been used as an alternative for similar long-term survival and better quality of life. Diabetes mellitus, heart disease, and chronic obstructive pulmonary disease were present in 6,10 , and 23 patients, respectively. Patient characteristics are listed in Table 1.

After surgery, the patients received chest CT and upper gastrointestinal X-ray at month 3 and 6 . At those same time points, related symptoms, such as dysphagia, heartburn, acid regurgitation, and nocturnal cough, were investigated. Dysphagia was graded according to a published method ( 0 = no dysphagia, $1=$ difficulty swallowing solid food,
$2=$ able to swallow soft food, $3=$ able to swallow liquids, and 4 = unable to swallow food). ${ }^{9}$ The frequency of heartburn, acid regurgitation, and nocturnal cough was graded according to the GerdQ criteria $(0=$ no related symptoms, $1=\geq 1$ day per week, $2=2-3$ days per week, and $3=4-7$ days per week). ${ }^{10}$

\section{Surgical Procedure}

Left thoracotomy was performed on 113 patients, and right thoracotomy with upper midline abdominal incisions was performed on 14 patients that had middle or upper esophageal carcinomas with potential involvement of the trachea and the azygos vein or enlarged upper right mediastinal lymph nodes. After mobilizing the esophagus and stomach, lymphadenectomy of the chest and upper abdomen were routinely performed. The gastric conduit was formed with a linear stapler (Ethicon TLC55; Johnson \& Johnson Medical, Shanghai, China), and an incision was made following the anterior border of the sternocleidomastoid muscle. The esophagus was mobilized and brought up to the level of the thyroid cartilage. The gastric conduit was brought out in an orthotopic position, with the lesser curvature facing right and the greater curvature facing left. The anastomotic portion of the esophagus and stomach was scored first. A row of 4-0 sutures was placed in a horizontal mattress fashion between the muscularis of the esophagus ( $3 \mathrm{~cm}$ up from the scored portion) and the musculoserosa of the stomach ( $2 \mathrm{~cm}$ down from the scored portion), and tied until the stapled anastomosis was completed. After the purse stitch of the esophagus was clamped, the specimen was excised. Ethicon SDH25 or SDH21 (Johnson \& Johnson Medical, Shanghai, China) were used for the circular stapled anastomosis. An anvil was placed into the esophagus and the stitch was tied carefully. A $2.5-\mathrm{cm}$ gastrotomy was made at the top of the gastric conduit, and the rod of the anvil was pushed into the gastric cavity through the scored portion. The anvil was then connected to the body of the stapler and fired. After checking the integrity of the anastomosis, a nasogastric tube and a nasointestinal tube were inserted. The redundant stomach was excised using a linear stapler. The posterior stitches were tightened to draw the stomach upward toward the esophagus. This procedure embedded the posterior of the anastomosis into the stomach cavity. A row of 4-0 interrupted sutures in a horizontal mattress fashion was completed over the remaining circumference of the esophagus and stomach, about $2 \mathrm{~cm}$ from the anastomosis. The anastomosis was then fully embedded and the stomach was folded upward around the remaining esophagus (Fig. 1). A rubber strip at the neck incision and a chest catheter were used for drainage. 


\section{Postoperative Treatment}

The drainage catheter and rubber strip were removed 3-4 days later, and enteral nutrition was administered through a nasointestinal tube after 3 days. One week after surgery, the gastric tube was removed and patients began taking food. Anastomotic leakage was considered to be resolved when digestive juice emerged at the incision or, after oral administration of methylene blue, blue liquid flowed out of the incision. The mean length of hospital stay was $18.5 \pm 1.9$ days and the mean stay in intensive care units was $2.2 \pm 1.0$ days.

\section{RESULTS}

\section{Surgical Results}

Hand-sewn end-to-side anastomosis was conducted in 2 patients whose stomachs were not long enough for the mechanical procedure. The anastomosis was not embedded in 2 patients because of high tension at the anastomosis site. The remaining patients successfully underwent the complete surgical procedure [123 of 127 (96.9\%)]. In 2 patients, anastomotic bleeding occurred, and after hemostasis was achieved by sewing and binding, the anastomosis was embedded. One patient showed a tear of the esophageal muscularis after gastroesophagostomy, which was embedded after repair.

\section{Postoperative Complications}

The total incidence of complications was 20 of 123 $(16.3 \%)$. Anastomotic leakage was seen in 4 patients (3.3\%) on days 4, 6, 8, and 10 after surgery, and after debridement and draining, the leakage stopped within 10-14 days. Other complications included pneumonia in 8 patients $(6.5 \%)$, arrhythmia in 5 patients $(4.1 \%)$, and injury of the recurrent laryngeal nerve in 3 patients $(2.4 \%)$. One patient $(0.8 \%)$ died as a result of acute respiratory failure induced by pneumonia. The relationship of leakage and clinical characteristics were tested by Chisquare tests; no special risk factor was found (Table 1).

\section{Postoperative Review}

After surgery, chest CTs and upper gastrointestinal $\mathrm{X}$-rays were reviewed for 123 patients at month 3 and 6 , respectively. The results noted that stricture $(<1.0 \mathrm{~cm})$ of anastomosis occurred in 12 patients, stricture $(<0.5 \mathrm{~cm})$ in 2 patients, and the total incidence of stricture was 14 of 123 $(11.4 \%)$. Severe stricture occurred in 2 patients and severe dysphagia occurred in 6 patients ( 8 of $123 ; 6.5 \%$ ); after
FIG. 1 Illustration of circular stapled anastomosis with embedded esophagus. a A row of 4-0 sutures was placed in a horizontal mattress fashion between the muscularis of the esophagus and the musculoserosa of the stomach, and the purse stitch of the esophagus was completed using a clamp. b The specimen was excised. The anvil was placed into the esophagus and the stitch was tied carefully. c A $2.5-\mathrm{cm}$ gastrotomy was made at the top of the gastric conduit, and the rod of the anvil was inserted into the gastric cavity. d The anvil was connected to the body of the stapler and fired. e The redundant stomach was excised using a linear stapler. $\mathbf{f}$ The posterior stitches were tied with attention to draw the stomach upward toward the esophagus. This procedure embedded the posterior of the anastomosis into the stomach cavity. A row of 4-0 interrupted sutures in a horizontal mattress fashion was completed over the remaining of circumference of the esophagus and stomach. $\mathbf{g}$ The anastomosis was fully embedded and the stomach was folded upward around the remaining esophagus

gastroscopic biopsy excluded cancer recurrence, these patients received dilatation treatment. A total of 18 dilatation sessions were conducted in all, equaling 2.25 times for each patient over an interval of 2 weeks. Symptoms relevant to gastroesophageal reflux are shown in Table 2. Six months after surgery, the incidence of dysphagia, heartburn, acid regurgitation, and nocturnal cough were 39 of $123(31.7 \%), 62$ of $123(50.4 \%), 11$ of $123(8.9 \%)$, and 16 of $123(13.0 \%)$, respectively.

\section{DISCUSSION}

In recent years, cervical esophagogastrostomy has been performed for many purposes. Two or three fields of lymph node dissection after subtotal esophagectomy could reduce the risk of cancer recurrence. ${ }^{11}$ Cervical esophagogastrostomy is a necessary component of transhiatal esophagectomy or minimal invasive surgery. ${ }^{12,13}$ Cervical esophagogastrostomy demands excellent surgical skill and the benefit of experience, due to the limited space at the neck and to protect the recurrent laryngeal nerve while freeing the upper portion of the esophagus. In a meta-analysis of randomized comparative studies, the incidence of postoperative anastomotic leakage and injury of the recurrent laryngeal nerve after cervical esophagogastrostomy were higher than those of intrathoracic anastomosis, but the sample sizes of the studies were small. ${ }^{14}$ For thoracic cancers in the middle and upper thirds of the esophagus, cervical esophagogastrostomy could be an inevitable choice for their radical resection.

The skilled application of anastomosis via circular stapler results in improved safety, comparable or superior to hand-sewn anastomosis. ${ }^{8,15,16}$ In a comparative study of 26 hand-sewn vs stapled anastomoses, postoperative anastomotic leakage and death shortly after surgery showed conflicting results between the methods, so benefits and drawbacks were unable to be determined. ${ }^{17}$ In recent years, mechanical or semi-mechanical side-to-side anastomosis 

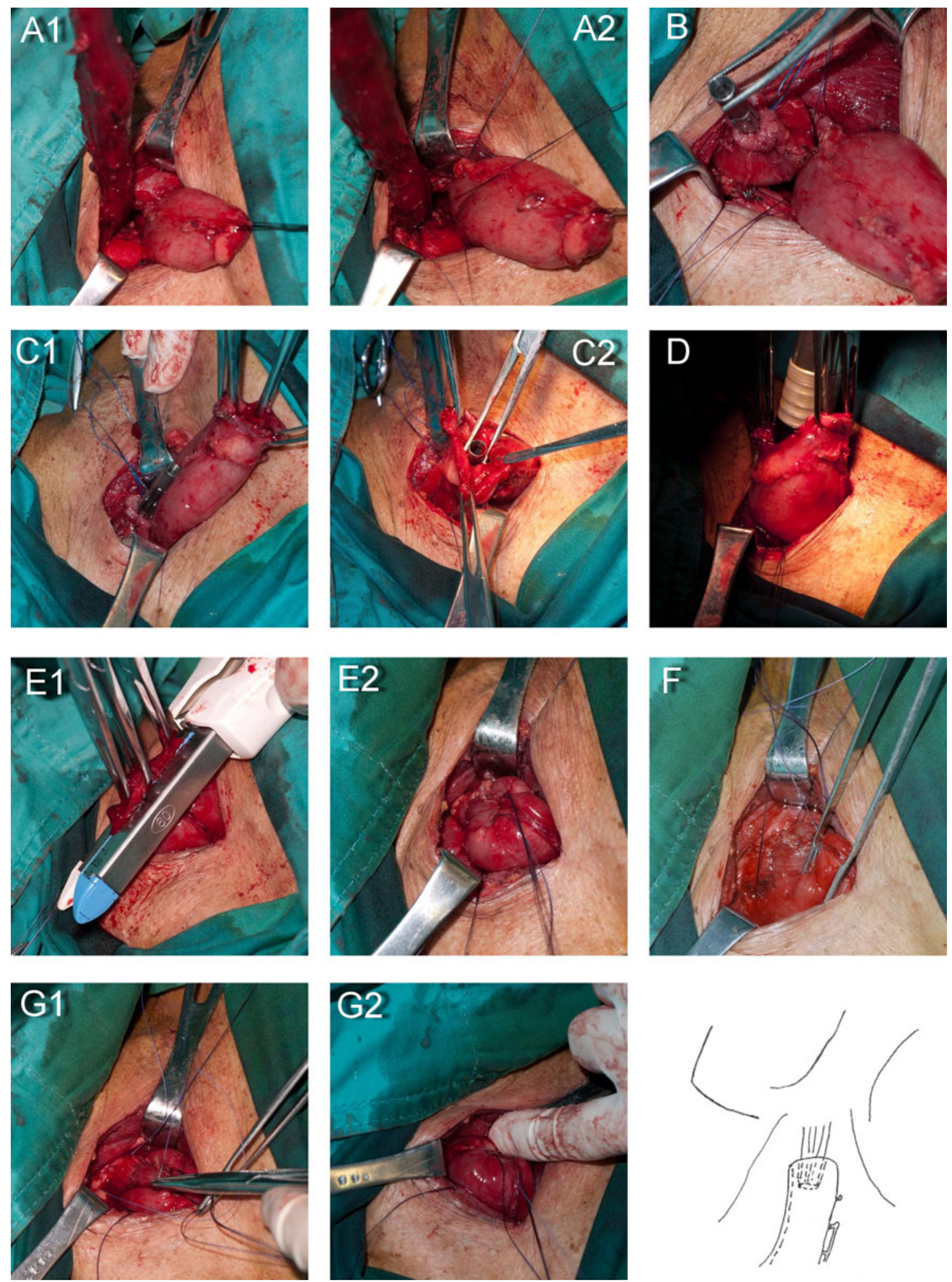

using a linear stapler has become popular, and the incidence of postoperative anastomotic leakage and anastomotic stricture have been shown to be reduced, but the longer residual end of the esophagus and gastroesophageal reflux remain concerns. ${ }^{18-20}$ Toh et al. reported a triangle anastomosis method in which an end-to-end method with good anastomoses between mucosa and blood vessels could reduce the incidence of postoperative anastomotic leakage and anastomotic stricture. ${ }^{21}$ Szücs et al. and Henriques et al. have also reported a lower occurrence 
of postoperative anastomotic leakage by a telescope-type anastomosis method, which places the remaining esophagus into the stomach cavity. ${ }^{22,23}$ However, both studies suffered from complex manipulations and the lack of controls.

At our hospital, we made two improvements based on the conventional stapled anastomosis with circular stapler. First, the anvil penetrated the stomach cavity, connected with the body of the stapler in the stomach cavity, and fired, requiring a shorter gastric conduit and reducing injury caused by pulling the stomach. Second, embedding the remaining esophagus and anastomosis in the stomach cavity facilitates healing and reduces the incidence of gastroesophageal reflux. A prerequisite for successfully performing these procedures is a piece of long- and thickenough tubular stomach. Otherwise, embedding the anastomosis is hard to complete. Initially, we thought that the diameter of the stapler was irrelevant to postoperative anastomotic leakage and anastomotic stricture, but the appropriate stapler should be selected according to the width of the esophagus. ${ }^{24}$ The anastomoses should be carefully checked before firing to prevent dislocation occurring between mucosa or muscular layers, and appropriate depth should be taken into account. The mobilized esophagus needs to be long enough to both embed and prevent the formation of stomach-esophagus angles after surgery, which could cause dysphagia. The incidence of postoperative anastomotic leakage in this study was $3.3 \%$; we thought that embedding the anastomosis could reduce the occurrence of postoperative anastomotic leakage. ${ }^{5-8,15,16,18-20}$

Currently, no recognized standard exists for anastomotic stricture because of the difficulty in evaluating distensibility. In a follow-up study of 9 patients who received semi-mechanical cervical esophagogastrostomy, no patients showed anatomic stricture, all patients experienced reflux laryngitis, and 5 patients showed subjective dysphagia, which was considered to be related to postsurgical dysfunction. ${ }^{19}$ We thought that dysphagia was not only related to the size of anastomosis, but also related to the angle and distensibility of anastomosis, as well as swallowing muscle function. In our study, we considered a $<1.0-\mathrm{cm}$ diameter of anastomotic stoma in barium meal tests as criteria: the incidence of stricture was $11.4 \%$ and dilatation was conducted in $6.5 \%$ of patients. As shown by the scoring of dysphagia, medium to severe dysphagia was accounted for 7 of 123 cases $(5.7 \%)$. These data were similar to those in the side-to-side anastomosis. ${ }^{5,8,18-20}$

Gastroesophageal reflux is one of the most common postoperative complications. ${ }^{3,25}$ We considered that embedding the remaining esophagus not only facilitates the healing of the anastomosis, but also plays an antireflux effect similar to the folding method at the bottom of the
TABLE 1 Clinical characteristics and the relation to leakage

\begin{tabular}{|c|c|c|c|}
\hline Characteristic & Value & $\begin{array}{l}\text { Ratio of } \\
\text { leakage }\end{array}$ & $P$ \\
\hline Age, $y$ & & & 0.33 \\
\hline Mean & $59.1 \pm 7.0$ & $<65,2.2 \%$ & \\
\hline Range & $38-72$ & $\geq 65,5.6 \%$ & \\
\hline Sex, M/F & $\begin{array}{c}79(62.2 \%) / 48 \\
(37.8 \%)\end{array}$ & $3.8 \% / 2.1 \%$ & 0.37 \\
\hline Tumor location & & & 0.60 \\
\hline Upper thoracic & $9(7.1 \%)$ & 0 & \\
\hline Middle thoracic & $81(63.8 \%)$ & $2.5 \%$ & \\
\hline Lower thoracic & $37(29.1 \%)$ & $5.4 \%$ & \\
\hline Cell type & & & 0.75 \\
\hline Squamous & $124(97.6 \%)$ & $3.2 \%$ & \\
\hline Adenocarcinoma & $3(2.4 \%)$ & 0 & \\
\hline Clinical TNM & & & 0.21 \\
\hline IA & $7(5.5 \%)$ & 0 & \\
\hline IB & $9(7.1 \%)$ & $11.1 \%$ & \\
\hline IIA & $17(13.4 \%)$ & $11.8 \%$ & \\
\hline IIB & $43(33.9 \%)$ & 0 & \\
\hline IIIA & $27(21.2 \%)$ & $3.7 \%$ & \\
\hline IIIB & $21(16.5 \%)$ & 0 & \\
\hline IIIC & $3(2.4 \%)$ & 0 & \\
\hline Comorbidity & & $\begin{array}{l}\text { (Without/ } \\
\text { With) }\end{array}$ & \\
\hline Diabetes mellitus & $6(4.7 \%)$ & $0 / 3.3 \%$ & 0.65 \\
\hline Heart disease & $10(7.9 \%)$ & $10 \% / 2.6 \%$ & 0.20 \\
\hline COPD & $21(16.5 \%)$ & $2.9 \% / 4.3 \%$ & 0.72 \\
\hline Surgical approach & & & 0.47 \\
\hline Left esophagectomy & $113(89.0 \%)$ & $3.5 \%$ & \\
\hline $\begin{array}{l}\text { Right } \\
\text { esophagectomy }\end{array}$ & $14(11.0 \%)$ & 0 & \\
\hline \multicolumn{4}{|l|}{ Hospital stay, d } \\
\hline Mean & $18.5 \pm 1.9$ & & \\
\hline Range & $10-31$ & & \\
\hline \multicolumn{4}{|l|}{ ICU stay, d } \\
\hline Mean & $2.2 \pm 1.0$ & & \\
\hline Range & $1-9$ & & \\
\hline
\end{tabular}

TNM tumor node, metastasis staging system, COPD chronic obstructive pulmonary disease, $I C U$ intensive care unit

stomach via the volume effect in the proximal stomach and compression of remaining esophagus by the multilayer stomach wall. Figure 2 shows the closed anastomosis at the horizontal position. Figure 3 shows an open anastomosis during a barium meal test and the compression of the esophagus by the upper portion of the stomach. Since it was hard to test acid in the remaining esophageal tissue, gastroesophageal reflux was evaluated according to subjective symptoms such as heartburn and acid regurgitation. The incidence of heartburn was 32.5 and $50.4 \%$ in months 3 and 6 after surgery, respectively, which was much higher 
TABLE 2 Postsurgery symptom scores of dysphagia and gastroesophageal reflux

\begin{tabular}{|c|c|c|c|c|c|c|c|c|}
\hline \multirow[t]{3}{*}{ Symptom } & \multicolumn{8}{|c|}{ Score } \\
\hline & \multicolumn{4}{|c|}{3 Months after surgery } & \multicolumn{4}{|c|}{6 Months after surgery } \\
\hline & 0 & 1 & 2 & 3 & 0 & 1 & 2 & 3 \\
\hline Dysphagia $^{a}$ & 93 & 26 & 5 & 3 & 88 & 32 & 7 & 0 \\
\hline Heartburn & 87 & 31 & 9 & 0 & 65 & 44 & 12 & 6 \\
\hline Acid regurgitation & 115 & 12 & 0 & 0 & 116 & 9 & 2 & 0 \\
\hline Nocturnal cough & 118 & 8 & 1 & 0 & 111 & 13 & 2 & 1 \\
\hline
\end{tabular}

${ }^{a}$ No patient with dysphagia had grade 4

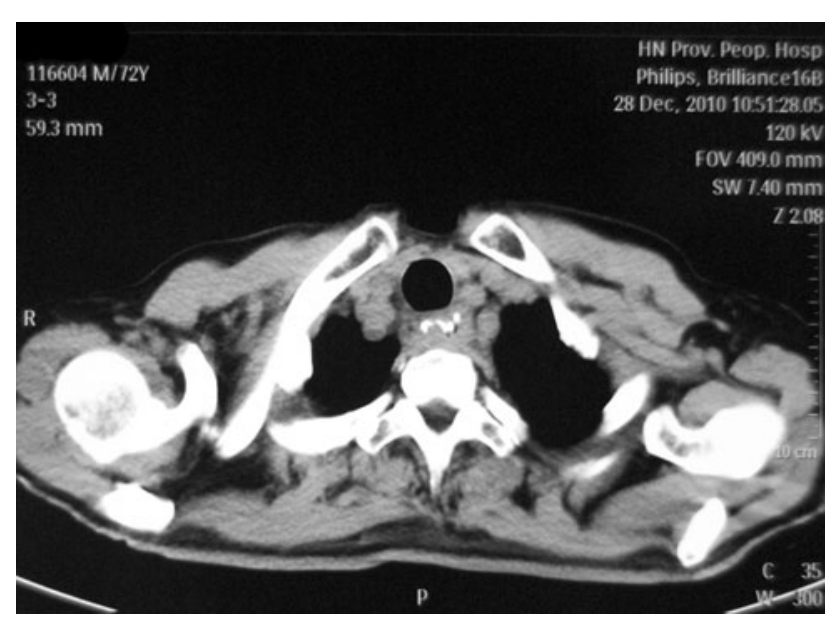

FIG. 2 Closed anastomosis at the horizontal position

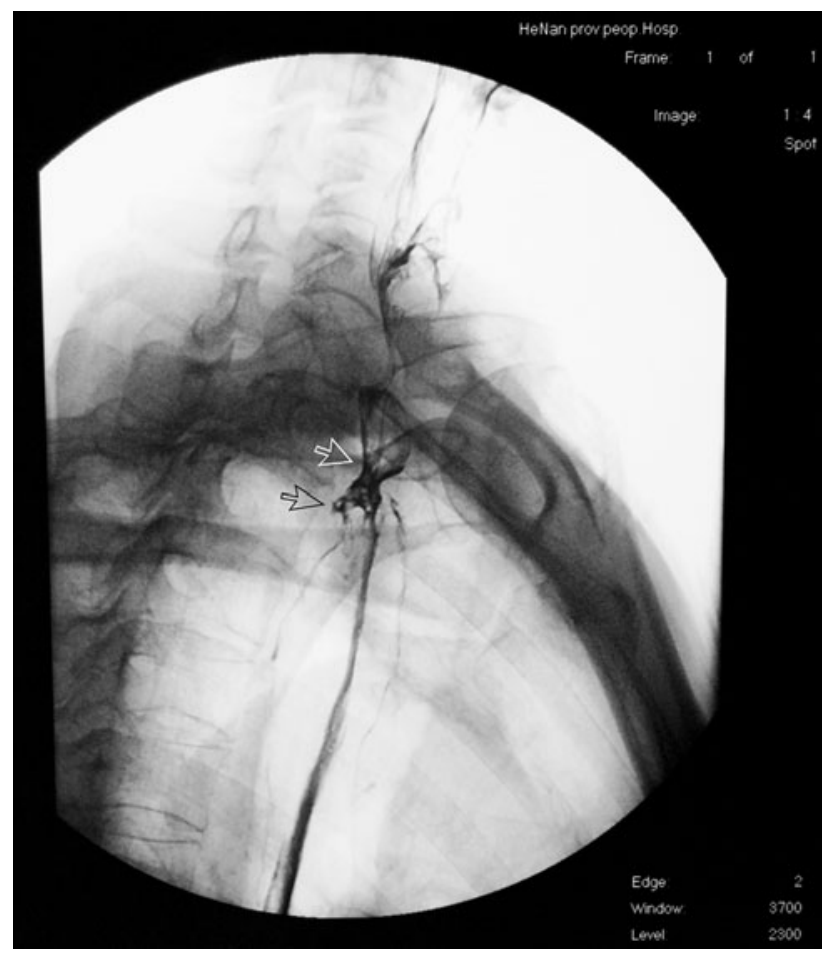

FIG. 3 Open anastomosis during a barium meal test (black arrow) and compression of the esophagus by the upper portion of the stomach (white arrow) than that of acid regurgitation (9.8 and $8.9 \%$, respectively). Although it was thought that the pyloroplasty could reduce the occurrence of reflux, we only dissociated the connective tissue around the pylorus instead of conducting pyloroplasty, and no severe duodenogastric reflux occurred. ${ }^{26}$. We thought that the gastric acid secretion of a nerve-free stomach could gradually recover over time, which was demonstrated by comparing the 3-and 6-month data, while no obvious change in acid regurgitation rate was observed, similar to other published results that indicated that embedding in the proximate stomach presented an antireflux effect. ${ }^{25}$

In conclusion, mechanical anastomosis with a circular stapler is easy and convenient, with just a one-time procedure. Embedding the remaining esophagus not only facilitates the healing of the anastomosis but can also reduce the incidence of gastroesophageal reflux. These results need to be confirmed in randomized controlled studies, and the antireflux effect should be further investigated.

Disclosure The authors declare no conflict of interest.

Open Access This article is distributed under the terms of the Creative Commons Attribution License which permits any use, distribution, and reproduction in any medium, provided the original author(s) and the source are credited.

\section{REFERENCES}

1. Kamangar F, Dores GM, Anderson WF. Patterns of cancer incidence, mortality, and prevalence across five continents: defining priorities to reduce cancer disparities in different geographic regions of the world. J Clin Oncol. 2006;24:2137-50.

2. Boone J, Livestro DP, Elias SG, Borel Rinkes IH, van Hillegersberg R. International survey on esophageal cancer: part I surgical techniques. Dis Esophagus. 2009;22:195-202.

3. Aly A, Jamieson GG. Reflux after oesophagectomy. Br J Surg. 2004;91:137-41.

4. Blackmon SH, Correa AM, Wynn B, et al. Propensity-matched analysis of three techniques for intrathoracic esophagogastric anastomosis. Ann Thorac Surg. 2007;83:1805-13.

5. Orringer MB, Marshall B, Iannettoni MD. Eliminating the cervical esophagogastric anastomotic leak with a side-to-side stapled anastomosis. J Thorac Cardiovasc Surg. 2000;119:277-88. 
6. Worrell S, Mumtaz S, Tsuboi K, Lee TH, Mittal SK. Anastomotic complications associated with stapled versus hand-sewn anastomosis. J Surg Res. 2010;161:9-12.

7. Laterza E, de' Manzoni G, Veraldi GF, Guglielmi A, Tedesco P, Cordiano C. Manual compared with mechanical cervical oesophagogastric anastomosis: a randomised trial. Eur J Surg. 1999;165:1051-4.

8. Hsu HH, Chen JS, Huang PM, Lee JM, Lee YC. Comparison of manual and mechanical cervical esophagogastric anastomosis after esophageal resection for squamous cell carcinoma: a prospective randomized controlled trial. Eur J Cardiothorac Surg. 2004;25:1097-101.

9. Stoller JL, Samer KJ, Toppin DI, Flores AD. Carcinoma of the esophagus: a new proposal for the evaluation of treatment. Can J Surg. 1977;20:454-9.

10. Jones R, Junghard O, Dent J, et al. Development of the GerdQ, a tool for the diagnosis and management of gastro-oesophageal reflux disease in primary care. Aliment Pharmacol Ther. 2009;30:1030-8.

11. Katayama A, Mafune K, Tanaka Y, Takubo K, Makuuchi M, Kaminishi M. Autopsy findings in patients after curative esophagectomy for esophageal carcinoma. J Am Coll Surg. 2003;196:866-73.

12. Osugi H, Takemura M, Higashino M, Takada N, Lee S, Kinoshita H. A comparison of video-assisted thoracoscopic oesophagectomy and radical lymph node dissection for squamous cell cancer of the oesophagus with open operation. Br J Surg. 2003;90:108-13.

13. Cooke DT, Lin GC, Lau CL, et al. Analysis of cervical esophagogastric anastomotic leaks after transhiatal esophagectomy: risk factors, presentation, and detection. Ann Thorac Surg. 2009;88:177-84.

14. Biere SS, Maas KW, Cuesta MA, van der Peet DL. Cervical or thoracic anastomosis after esophagectomy for cancer: a systematic review and meta-analysis. Dig Surg. 2011;28:29-35.

15. Zhou NK, Liu X, Liang CY, Tian XD, Liu Y, Yuan Y. The application of stapling device in surgery of carcinoma of the esophagus and cardia. Zhonghua Yi Xue Za Zhi. 2006;86:1450-2.
16. Pines G, Buyeviz V, Machlenkin S, Klein Y, Laor A, Kashtan H. The use of circular stapler for cervical esophagogastric anastomosis after esophagectomy: surgical technique and early postoperative outcome. Dis Esophagus. 2009;22:274-8.

17. Kim RH, Takabe K. Methods of esophagogastric anastomoses following esophagectomy for cancer: a systematic review. J Surg Oncol. 2010;101:527-33.

18. Singh D, Maley RH, Santucci T, et al. Experience and technique of stapled mechanical cervical esophagogastric anastomosis. Ann Thorac Surg. 2001;71:419-24.

19. Koh P, Turnbull G, Attia E, LeBrun P, Casson AG. Functional assessment of the cervical esophagus after gastric transposition and cervical esophagogastrostomy. Eur J Cardiothorac Surg. 2004;25:480-5.

20. Williams VA, Watson TJ, Zhovtis S, et al. Endoscopic and symptomatic assessment of anastomotic strictures following esophagectomy and cervical esophagogastrostomy. Surg Endosc. 2008;22:1470-6.

21. Toh Y, Sakaguchi Y, Ikeda O, et al. The triangulating stapling technique for cervical esophagogastric anastomosis after esophagectomy. Surg Today. 2009;39:201-6.

22. Szücs G, Toth I, Gyani K, Kiss JI. Telescopic esophageal anastomosis: operative technique, clinical experiences. Dis Esophagus. 2003;16:315-22.

23. Henriques AC, Godinho CA, Saad R Jr, et al. Esophagogastric anastomosis with invagination into stomach: new technique to reduce fistula formation. World J Gastroenterol. 2010;16:5722-6.

24. Yendamuri S, Gutierrez L, Oni A, et al. Does circular stapled esophagogastric anastomotic size affect the incidence of postoperative strictures? J Surg Res. 2011;165:1-4.

25. Aly A, Jamieson GG, Watson DI, Devitt PG, Ackroyd R, Stoddard CJ. An antireflux anastomosis following esophagectomy: a randomized controlled trial. J Gastrointest Surg. 2010;14:470-5.

26. Yuasa N, Sasaki E, Ikeyama T, Miyake H, Nimura Y. Acid and duodenogastroesophageal reflux after esophagectomy with gastric tube reconstruction. Am J Gastroenterol. 2005;100:1021-7. 